A Lee

O n 5 July 2003, the WHO removed Taiwan from its list of areas with recent local transmission of SARS meaning that all known chains of person to person transmission of the severe acute respiratory syndrome (SARS) virus have now been broken. ${ }^{1}$ However, the WHO executive director for communicable diseases advised that public health should not let down its guard, as more cases could still surface somewhere in the world. ${ }^{1}$ It is therefore an important public health issue whether the SARS epidemics will recur.

If you had a crystal ball to view the future, this question would be answered. We would make some prediction based on the epidemiological triangle (fig 1) recognising the three main factors-agent, environment, and host in the pathogenesis of disease. ${ }^{2}$ If we can control any two of the main factors, we would prevent the occurrence of a communicable diseases.

Coronavirus has been identified in playing an aetiological part of SARS. ${ }^{3}$ A lot of work has been done to understand the genome of the virus that would lead to development of vaccine and treatment, but time is needed for such development. To prevent the recurrence of epidemics we should look at the host and environmental factors.

The agent must be capable of infecting the host for infection to develop. This depends on whether the environment is favourable for its survival and transmission, and also the susceptibility of the host. The susceptibility of the host depends on its ability to fight off the infection, which can be a disease specific defence mechanism such as vaccine, or non-specific defence mechanism. The ability of non-defence mechanism to fight off infectious disease will depend on the host's general health status, nutritional status, age, coexisting chronic illness, etc. If you have a population that is healthy, fit, and well nourished, the chance of infection would be low.

Epidemics are an increase in the frequency of occurrence of a disease in a population above its baseline level for a specified period of time. To calculate this, estimate the basic reproductive number that is defined as the expected number of new infectious hosts that one infectious host will produce during the period of infectiousness in a population that is susceptible. It depends on number of contacts per unit time, transmission probability, and duration of infectiousness. Apart from infectivity of the agent and host suscept-

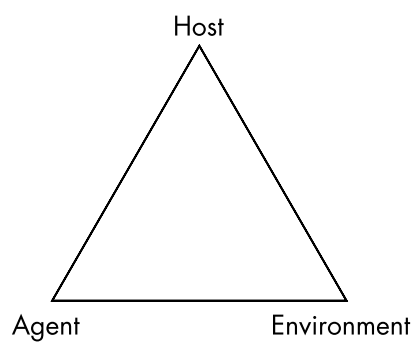

Figure 1 The epidemiological triangle.

ibility, transmission probability also depends on environmental factors. SARS is spread by droplet transmission. If the population has developed good personal hygienic practice to prevent the spread of droplet infection, and creates a clean and hygienic environment minimising the chance of survival of infectious disease agents, the transmission probability will be low. If the suspected SARS cases can be screened early and isolated, the number of contacts per unit time will be fewer and the duration of susceptible hosts exposed to suspected cases will also be short. Therefore environment improvement and good infectious disease control measures are the key factors to prevent an epidemic in the autumn of 2003.

Before a breakthrough in treatment and vaccine development, a SARS epidemic can easily recur if the population is unhealthy and has poor hygienic practices, and where there is inadequate infectious control measures and a poor living environment. The Hong Kong SAR government has taken measures to improve the environment, and also to promote community action and partnership in improving the health and hygiene. It really depends whether these initiatives can be sustained as to whether the epidemic is less likely to recur.

\section{REFERENCES}

1 WHO. Taiwan, China: SARS transmission interrupted in last outbreak area. Communicable Disease Surveillance and Response 2003;update 96, 5 Jul. http://www.who.int/csr/ don/2003_07_05/en/.

2 Mansner JS, Kramer S. Epidemiology: an introductory text, 2nd edn. Philadelphia: Saunders, 1985.

3 Ksiazek TG, Erdman D, Goldsmith C, et al. A novel coronavirus associated with severe respiratory syndrome. N Engl J Med 2003;348:1953-66.
A S M Abdullah, Department of Community Medicine, The University of Hong Kong, 5/F Academic Block, 21 Sassoon Road, Pokfulam, Hong Kong; asma@hkucc.hku.hk

\title{
Virus pathogens suggest an autumn return
}

\section{A S M Abdullah}

S ARS is one of the deadly new emerging infectious diseases identified in the $21 \mathrm{st}$ century. Since its emergence in November
2002, SARS has created public panic and raised many issues among healthcare workers and policy makers around the world. Although 
healthcare communities together with public vigilance around the world seem to have halted the SARS outbreak, at least for the time being, the question remains to be answered is whether the infection will reappear? I believe if SARS follows the pattern of other

\section{Policy implications}

- Greater vigilance about health and hygiene and the open sharing of medical information should be the norm in future.

- In the absence of any effective vaccine or treatment, the only way to combat SARS is to limit its spread.

- If SARS does return in autumn its epidemiology could be different.

respiratory viruses, it is probable that it will reappear next autumn during the influenza season. I have the following explanations in support of my opinion.

The causative agent of SARS is a novel coronavirus-a virus of the corona family. About one third of all common colds are caused by viruses from the same family and these show a winter and spring seasonality. ${ }^{1}$ The emergence of SARS outbreak in China and Hong Kong during the influenza season (December-March) suggests that possible common environmental factors may influence transmission. Some human pathogens such as influenza, measles, and rotavirus follow a cyclical pattern, waxing in colder and drier months and waning when weather turns warmer, ${ }^{2}$ which may also be the case for the SARS virus. While the importance of effective quarantine and preventive measures cannot be ignored, the decline in the SARS prevalence with increasingly warmer weather supports that seasonality may be a contributing factor. This may explain the limited spread of the SARS virus in the rural areas of Guangdong, which has only basic medical facilities and lower public preparedness. For instance, environmental temperature may influence the trends of SARS outbreak. Outbreaks of respiratory syncytial virus infections were associated with higher environmental temperature, lower relative humidity, and higher maximum day to day temperature variations. ${ }^{3}$ During the Amoy garden outbreak in Hong Kong, external temperature ranged between $18-22^{\circ} \mathrm{C}$, which has been proposed as permissive temperature enabling transmission of the SARS coronavirus. It is unconvincing that Vietnam and Guangzhou controlled SARS by better medical facilities and hygienic standard. Anecdotal reports suggest that the changes in temperature might have limited the outbreak of SARS in Vietnam and Guangzhou earlier than Hong Kong. The wider

\section{Key point}

- If SARS follows the pattern of other respiratory viruses, it is probable that it will reappear next autumn during the influenza season.

use of heaters in Toronto and air conditioning in Hong Kong and Singapore, usually to keep the room temperature within $18-22^{\circ} \mathrm{C}$, might have contributed to the long lasting outbreak in these developed cities. It would be useful to examine the relationship between temperature change and the occurrence of SARS in the future.

Consistent with other infectious diseases, ${ }^{2}$ changes in atmospheric conditions, the prevalence of virulence of the pathogen and the behaviour of the host could also contribute to the recurrence of SARS. It is possible that the virus is being slowly transmitted among people who remain asymptomatic or the virus is surviving in the environment and will reappear when favourable conditions return. Although the source of the coronavirus remains to be confirmed, civet cats and other wild animals sold in food markets in southern China are believed to be the source. If confirmed the animals will be a reservoir in ready contact with humans that could initiate a second SARS epidemic.

Finally, whether or not SARS reappears, lessons learned from the recent outbreak such as greater vigilance about health and hygiene and the open sharing of medical information should be a norm in future. In the absence of any effective vaccine or treatment, the only way to combat SARS is to limit its spread. We should also be aware that if SARS does return in autumn its epidemiology could be different. In the recent outbreak, most of the SARS cases with the exception of Amoy Garden outbreak in Hong Kong were confined to the healthcare workers indicating limited community spread. Given the high case fatality rate, if the rate of transmission should increase in the community the consequences could be devastating.

\section{ACKNOWLEDGEMENTS}

I thank Dr G N Thomas (Department of Community Medicine, The University of Hong Kong) for his helpful comments on this paper.

\section{REFERENCES}

1 Chilvers MA, McKean M, Rutman A, et al. The effects of coronavirus on human nasal ciliated respiratory epithelium. Eur Respir J 2001;18:965-70.

2 Chew FT, Doraisingham S, Ling AE, et al. Seasonal trends of viral respiratory tract infections in the tropics. Epidemiol Infect 1998;121:121-8.

3 Dowell SF. Seasonal variation in host susceptibility and cycles of certain infectious diseases. Emerging Infect Dis 2001 ; 7:369-74. 


\section{Debate Identification and control are the priority}

\section{G Rezza}

G Rezza, Istituto Superiore di Sanità, Viale Regina Elenà 299 00161 Rome, Italy; g.rezza@iss.it
T he global crisis brought about by the sudden appearance of the severe acute respiratory syndrome (SARS) seems to be over. The rapid and efficient control measures triggered by the alert launched by the World Health Organisation (WHO) have lowered the coefficient of reproduction to below one in all of the outbreak areas, and as of this writing more than 20 days have passed since the last case of SARS was reported. None the less, we do not know whether or not SARS has been eradicated. It is possible that SARS-CoV continues to be transmitted at a low level among humans or that it persists in an animal reservoir or in another environmental source and that it could cause outbreaks once certain conditions are again met. This uncertainty derives from a lack of knowledge on several aspects of the disease, knowledge that could aid in defining the dynamics of the epidemic and in formulating projections. In particular, the following questions still need to be answered: (1) Is the occurrence of SARS seasonal?; (2) What is the clinical spectrum of SARS-CoV infection and for how long do patients who have recovered continue to excrete SARS-CoV in respiratory secretions or stool?; and (3) Does an animal cycle or an environmental source of infection exist?

As is the case with infections caused by other human coronaviruses, such as 229E, the occurrence of SARS could be seasonal, peaking in late winter. If so, then the interruption in the chain of transmission is only temporary, and the virus is still present in human beings. We know that other severe viral infections, such as Ebola and Lassa fever, can be asymptomatic, although persons with asymptomatic infection are not necessarily able to transmit the infection to other susceptible hosts. With regard do SARS, the few studies that have been conducted have shown no evidence of infection (that is, RNA sequences or seroconversion) among healthy contacts of SARS cases or other SARS-free people. ${ }^{12}$ To determine whether or not SARS-CoV can be excreted in the long term, possibly in faeces, we need data from the long term follow up of persons who have recovered, yet these data are not available. Moreover, misdiagnosis or under reporting, especially in cases of atypical or mild clinical presentation, may occur, and this could maintain the chain of transmission at an undetectable level.

As found with Ebola, it is possible that SARS$\mathrm{CoV}$ continues to circulate in an animal reservoir and that under certain conditions it may again cross into the human population. A coronavirus similar to SARS-CoV but with an extra stretch of 29 nucleotide bases has been found in palm civets and a racoon dog. ${ }^{3}$ However, as viruses tend not to gain stretches when they jump across species, it is very unlikely that SARS-CoV moves from humans back to animals and then back to humans. None the less, SARS-CoV or similar viruses might survive in another animal reservoir and cross the barrier again, yet we cannot predict when. Finally, whether other environmental reservoirs exist remains unknown.

With these caveats in mind, it can be said that predicting the recurrence of SARS is quite difficult. Our priority thus becomes that of strengthening the capacity to identify and control future outbreaks of the disease.

\section{REFERENCES}

1 Drosten C, Gunther S, Preiser W, et al. Identification of a novel coronavirus in patients with severe acute respiratory syndrome. N Engl J Med 2003:348:1967-76.

2 Peiris JSM, Lai ST, Guan Y, et al. Coronavirus as a possible cause of severe acute respiratory syndrome. Lancet 2003:361:1319-25.

3 Cyranoski D, Abbott A. Virus detectives seek source of SARS in China's wild animals. Nature 2003:423:467.
T W Wong, Department of Community and Family Medicine, The Chinese University of Hong Kong,

$4 / F$ School of Public Health, Shatin, NT, Hong Kong;

twwong@cuhk.edu.hk

\section{Sporadic contact with unfamiliar source makes epidemic unlikely T W Wong}

S evere acute respiratory syndrome (SARS) has caught the world off guard. The outbreak first started in November 2002 in Guangdong province of China and reached Hong Kong in February 2003. It then spread quickly through jet travel to various countries. After several months of menace to healthcare workers and the community, SARS died down as suddenly as it appeared, sparking a question whether it will return this winter or later. To help us make an "educated" guess, we have to consider several factors.

\section{SEASONALITY}

Many viral respiratory diseases demonstrate a distinct seasonal pattern. However, the underlying reasons for this seasonality have not been fully understood. Influenza peaks in the winter months in temperate countries, which has generated hypotheses ranging from closer indoor 


\section{Key points}

- Seasonality is a common phenomenon of viral respiratory infections.

- The as yet unidentified source of infection makes it possible for SARS to recur, even in the absence of a subclinical carrier of the virus.

- Effective public health measures should limit the scale and the spread of any future epidemic.

personal contact in winter to the more intriguing relation with latitude and solar radiation. ${ }^{1}$ A similar pattern has been observed with respiratory syncytial virus infections, with an inverse relation with temperature and sunshine. Whether these seasonal changes affect the activity of the virus or host behaviour is still unclear. It is reasonable to assume that the SARS coronavirus would demonstrate a similar seasonal pattern. In winter months, there might be an increased probability of transmission between personal contacts and a change in dietary habits* among the Cantonese (people of Guangdong province origin, including the majority of the Hong Kong population).

\section{SOURCE OF INFECTION}

The source of the SARS coronavirus has not yet been determined, but preliminary viral isolation studies in Shenzhen (right across the border of Hong Kong) have suggested wildlife like the masked palmed civet cat and the raccoon dog might have been the natural host. ${ }^{2}$ This was further supported by the presence of antibodies to SARS virus among wild animal handlers. It is not clear if seropositive animal handlers are infectious and might constitute a potential source of infection. Another important source for a future epidemic would be the patient with subclinical infection. However, it is not certain whether this entity exists. Serosurveys among over 800 asymptomatic healthcare workers in the Prince of Wales Hospital (where the first outbreak of SARS occurred) in Hong Kong failed to demonstrate SARS antibodies (titre $\leqslant 1$ in 40 ) by the indirect immunofluorescence assay. As the SARS coronavirus is a new virus, it is unlikely to have adapted itself to produce a subclinical

*Dietary preferences like eating exotic wildlife originate from a traditional Chinese folk belief that extra nutrition is needed-rich in wildlife meat-in cold seasons.
Policy implications

- Vigilant disease surveillance and rapid and effective public health response are vital in the control of SARS, if and when it returns.

infection, or for the human host to develop sufficient immunity to avert a full clinical illness. Hence, if SARS returns, the source of infection should be similar to that which caused this epidemic, probably some form of wildlife that came into contact with its human handler, thereby transmitting the SARS virus to the handler.

\section{PUBLIC HEALTH RESPONSE}

The last factor that influences the spread of a future SARS epidemic is the effectiveness of the global disease surveillance system and the efficiency of the public health response. Early case detection and isolation appear to be the most effective control measures. Home quarantine, personal protection measures, and voluntary restriction of social activities adopted by the community are of unsure value, but should theoretically interrupt disease transmission. International travel restrictions and surveillance of febrile travellers might limit global spread. There are good reasons for optimism, as the worst affected countries and regions in this epidemic have learnt the lesson quickly and should be able to put these measures in place before the epidemic gathers force. How to prevent the amplification of SARS in the hospital, through better hospital design, more adequate isolation facilities, and better training and protection of healthcare workers remains a problem in less developed countries.

\section{WILL SARS COME BACK?}

Without identifying (and hence eliminating) its source and reservoir, it is possible that SARS will make a comeback, even in the absence of a subclinical human carrier. However, the transmission of the virus from a (presumed) wild animal host to a susceptible human host is a stochastic event and the probability of a large scale epidemic is low.

\section{REFERENCES}

1 Hope-Simpson RE. The role of season in the epidemiology of influenza. J Hyg 1981;86:35-47.

2 Cyranoski D, Abbott A. Virus detectives seek source of SARS in China's wild animals. Nature 2003;423:467.

\section{Future outbreaks will be less dramatic L Y Hsu, N I Paton}

$\mathrm{H}$ istory is replete with accounts of novel viral epidemics. Many of these viruses, like the human immunodeficiency virus or influenza virus, have become endemic. Others, like
Ebola or Sin Nombre viruses, cause outbreaks when conditions facilitating their transmission from animal to human hosts (still unknown in the case of Ebola) are met. Some epidemics, such
Debate
L Y Hsu, Department of Internal Medicine, Singapore General Hospital, Singapore N I Paton, Communicable Diseases Centre, Tan Tock Seng Hospital, Singapore

Correspondence to: Dr L Y Hsu, Department of Internal Medicine Singapore General Hospital, Outram Road, Singapore 169608; liyang_hsu@yahoo.com 


\section{Debate}

as the one caused by Nipah virus in Malaysia and Singapore, have occurred only once, but the potential for recurrence exists because of the presence of animal reservoirs. Even when diseases are apparently eradicated, the possibility of unnatural recurrences because of acts of bioterrorism must be considered. Although smallpox appeared to be the greatest threat at the beginning of 2003, the proven chaos and economic damage created by severe acute respiratory syndrome (SARS) may have made this novel coronavirus a particularly attractive agent in this regard.

At the time of writing, it seems likely that SARS coronavirus can be eradicated from humans. Chains of transmission have been broken worldwide, and the final case reported was isolated in Taiwan on 15 June 2003. ${ }^{1}$ The probability of a recurrence therefore depends on the existence of an animal reservoir. On a search of wildlife markets in Guangdong, investigators have isolated a remarkably similar virus in both the masked palm civet cat (Paguma larvata) and the raccoon dog (Nyctereutes procyonoides). ${ }^{2}$ One of the index cases of the Guangdong outbreak was a chef who specialised in wild game. These findings, together with the known propensity of coronaviruses to cause infections in animals, suggest that an animal reservoir could indeed exist. Although the precise circumstances that led the virus to be transmitted to humans are not known, these are unlikely to be so extreme that future replication will be impossible. Given the current crackdown on unsanctioned capture and sale of wildlife in southern China, and the heightened awareness of the disease in that region, we feel that SARS is unlikely to recur in autumn 2003.

However, we also believe that subsequent outbreaks will occur, although these will be smaller and more easily contained if the lessons learnt from this epidemic are not forgotten. The heterogeneity of the clinical presentations of SARS is now well known, ${ }^{3}$ and there is consequently less chance of missing atypical cases that could go on to amplify the outbreak. Nosocomial cases constituted the bulk of the epidemics in all countries involved, and the institution of strict infection control measures has been pivotal in ending these outbreaks. All hospitals should now have protocols in place, and the delay between the appearance of a new SARS case and implementation of appropriate measures should be greatly reduced in the future. The diagnostic tests we now have available, although of limited practical use in managing an established outbreak (in our experience), may prove advantageous in the early confirmation of a new outbreak and may therefore also accelerate the adoption of response measures. The lack of an antiviral drug of verified efficacy and of a vaccine underscores the need for continued vigilance and preparedness to ensure that future outbreaks are contained as effectively as the one we have just overcome.

\section{REFERENCES}

1 World Health Organisation. SARS outbreak contained worldwide. http://www.who.int/mediacentre/releases/ 2003/pr56/en/(accessed 6 Jul 2003).

2 Bonn D. Wild animals could be source of SARS. Lancet Infect Dis 2003;3:395.

3 Fisher D, Lim TK, Lim YT. Atypical presentations of SARS Lancet 2003:361:1740.

\section{Vigorous controls minimise risk}

\section{S H Lee}

A fter a trying period of three months battling against the severe acute respiratory syndrome (SARS) epidemic, Hong Kong was ultimately removed from the list of infected areas by the WHO on 23 June 2003. As we were struggling along our path against this epidemic, the disease unfolded its characteristics. It was a disease that would bring rapid progression to respiratory failure, it posed greatest threat to the health workers, the origin of its aetiological agent coronavirus was not firmly established, hypothesised to be of animal origin, there was no formalised standard way of treatment, no rapid method of diagnosis, and the disease was spreading rapidly internationally within Asia and to Europe and North America.

After introducing vigorous control measures and with the strong commitment of the government and the support of the healthcare workers and the community, assisted by experts from the WHO, the SARS epidemic in Hong Kong was brought under control after this hectic period of three months from March to June 2003.
After the victory over the epidemic, Hong Kong has quickly started its post-SARS activities to revitalise its economy, to strengthen its surveillance measures both locally and regionally including the Pearl River Delta region in mainland China, step up research activities to establish the origin of the aetiological agent, and to develop vaccines and new rapid diagnostic methods.

Three committees have been set up by government to take up specific responsibilities after the SARS epidemic. One committee was responsible for the overall cleansing campaigns and environmental improvements of the housing estates in the city. The second committee was responsible for drawing up plans and programmes to revitalise the economy of the city including tourism, trade, and employment. The third committee undertook to draw up strategies and plans to promote community involvement and partnership in improving the physical, social, and economic environments of the city. ${ }^{1}$

In June 2003, the government announced that an expert committee would be set up to review the 
management of the recent SARS outbreak in Hong Kong. The objective of the committee was to identify lessons to be learnt, and to make recommendations on areas of improvements to better prepare the system for any future outbreaks. Members of the committee comprise both overseas and local experts. The committee started its work at the beginning of July 2003. It will submit its report to the government by September 2003.

In the meantime, considerable amount of activities after the SARS epidemic have started. These include the introduction of a "Hygiene Charter" to promote personal and environmental hygiene in all settings such as schools, communities and workplaces, the mounting of city wide cleansing campaigns, joint meetings of pubic health workers from Hong Kong, Macau and Guangdong Province in mainland China to strengthen communication and expand the surveillance system on SARS and other major infectious diseases, and joint research activities on SARS between the academic institutions in Hong Kong and Guangzhou. Additional resources have been allocated by the government to promote research, to improve the ward conditions in hospitals including their isolation and ventilation facilities, to set aside specially designed bed facilities for the isolation and treatment of infectious disease patients, and to establish outbreak management plans in hospitals to deal with future outbreaks.

In the coming months, Hong Kong will continue case identifications through surveillance, to determine whether the infection is endemic or seasonal, or whether it has disappeared from human populations. It will continue collaboration with mainland China, particularly Guangdong Province in studies to identify animal reservoir and risk factors for transmission to humans. This measure is necessary to manage the risk and prevent future outbreaks. It will continue to participate in major WHO networks of global surveillance for influenza and other infectious diseases. This step is necessary in order to identify major emergence of new influenza strain or other infection of international importance. ${ }^{2}$

These activities could well demonstrate that Hong Kong will continue to maintain a high level of vigilance and preparedness to deal with another SARS epidemic should it occur in future. In the light of these observations, it is anticipated that in the coming autumn of 2003, Hong Kong is unlikely to face another SARS epidemic of such a magnitude as the last one. However, as respiratory illness is usually more prevalent in the cold season and bearing in mind the large volume of people travelling between Hong Kong and China, some sporadic cases of atypical pneumonia may occur, but it is not expected that another SARS outbreak will come again in such a short time.

\section{REFERENCES}

1 Lee SH. The SARS epidemic in Hong Kong-what lessons have we learned? J R Soc Med (in press).

2 Heymann D. Severe acute respiratory syndrome (SARS): global alert, global response. WHO, 15 Jun 2003.

\section{Avoid complacency-prepare for the worst J J Y Sung}

S evere acute respiratory syndrome (SARS) is the first severe and readily transmissible disease that has emerged in the $21 \mathrm{st}$ century. It has a unique capacity of spreading quickly in hospitals and clinics affecting thousands of healthcare workers in a short period of time. The booming of international air travel in recent decades has also empowered the infection to be quickly spread across the continents. In March 2003, one infected tourist from China checked into a hotel in Hong Kong and spread the infection to eight countries within two weeks. By July 2003, 32 countries were involved and 8439 patients were infected around the world. Although the WHO has announced that the initial crisis is over, we should not be complacent. There is, in my personal view, a high possibility that the epidemic will come back during the winter. This prediction is based on three reasons. Firstly, despite much effort contributed by virologists and molecular biologists, we are still not sure of the source of the infection. Early reports suggested that SARS-CoV resembled the bovine coronavirus or mouse hepatitis virus. Yet, after the sequence of the whole viral genome was completed, it becomes clear that the SARS-CoV is a distinctly new pathogenic strain that does not arise from a simple recombination of known existing strains. ${ }^{1}$ But, we are still not sure where it comes from. Secondly, serological studies have reviewed that asymptomatic or subclinical infection of SARS$\mathrm{CoV}$ is uncommon. We examined 674 healthcare workers at the Prince of Wales Hospital, where the first hospital outbreak occurred in Hong Kong, among them $43 \%$ had direct contact with SARS patients. None of them had IgG antibody to SARS-CoV. ${ }^{2}$ Similar studies conducted in China confirmed that asymptomatic carriers are few. This implies that immunity to SARS-CoV in the community will develop slowly. Thirdly, although RNA viruses are known to mutate more readily than DNA viruses, genomic studies of SARS-CoV including strains isolated from Singapore, Hong Kong, Guangdong, and Beijing showed a remarkable genetic conservation of the virus since the outbreak was first started in November 2002. ${ }^{3}$ Unlike most other infectious agents transmitted by direct person to person contact, the SARS-CoV is unlikely to mutate to a benign infection and attenuated symptoms. Without herd immunity and attenuation of the
Debate
J J Y Sung, Prince of Wales Hospital, Shatin, New Territories, Hong Kong; joesung@cuhk.edu.hk 


\section{Debate}

virus, when the next epidemic comes, one would still expect to have large scale outbreak with severe symptoms. What can we do to prepare for the come back? It will be some time before a vaccine can be developed, if it could ever be developed. We should continue to search the source of the virus and study the mode of transmitting the disease. We should maintain our high vigilance of the infection. When we see suspected cases, we should implement isolation, quarantine, and cohorting measures. Developing a rapid diagnostic test, probably polymerase chain reaction based, which can differentiate SARS from other atypical pneumonia at an earliest possible stage of the disease would be instrumental in applying these measures successfully. Don't let down your guard, the battle is not over yet!

\section{REFERENCES}

1 Marra MA, Jones SJM, Astell CR, et al. The genome sequence of the SARS-associated coronavirus. Science 2003;300:1388-404.

2 Chan PKS, Ip M, Ng KC, et al. Sero-prevalence of severe acute respiratory syndrome (SARS)-associated coronavirus infection among health care workers after a major outbreak of SARS in a regional hospital. Emerging Infect Dis (in press).

3 Ruan Y, Wei CL, Ee LA, et al. Comparative full-length genome sequence analysis of 14 SARS coronavirus isolates and common mutations associated with putative origins of infection. Lancet 2003;361:1779-85.

\section{Out of the woods or in the eye of the storm? R A Chotani}

A s of 4 July 2003 there have been 8439 cumulative cases of SARS-CoV globally, which were identified in over 28 countries resulting in 812 deaths (a case fatality proportion of $9.6 \%) .{ }^{1}$ With the most recent date of onset of probable cases of SARS in Taiwan and Canada 16 and 19 days ago respectively, in four days' time 20 days (two incubation periods) would have passed, the time required for the chain of human to human transmission to be considered broken. ${ }^{2}$ With the rapid decline in new probable cases there is jubilation that perhaps the scourge has been conquered. Fear prevails among researchers and scientists that the virus will persist in humanity indefinitely and will reemerge with a vengeance during the winter of 2003.

The reason for this fear is valid and based upon past experiences with the influenza virus. Comparisons have been made between SARS and influenza, in particular the 1918-19 Spanish flu pandemic. The outbreak started in the winter of 1918 and fizzled off during the summer, reemerging with a vengeance in 1919 resulting in millions of deaths globally. Eerie similarities exist between these two mutually exclusive viruses. The 1918-19 influenza strain, like the SARS-CoV, was highly transmissible, virulent, had moved from animals to humans, shared the same seasonal pattern, and the main source of transmission was through droplets. Moreover, there was no effective antiviral or vaccine. One of the advantages during the SARS-CoV epidemic was that the incidence of influenza globally was comparatively low, making it easier to identify SARS. The good news is that the virus is transmitted through large virus laden droplets (travel distance one to two metres) compared with influenza, which is lighter, ${ }^{3}{ }^{4}$ the disease can be identified, the genetic code of the virus is being deciphered, and it is seasonal and stays quiet during summer, which provides the time to prepare.
Lessons learned from the SARS-CoV outbreak clearly demonstrate that delayed reporting of unusual disease events can lead to a global catastrophe; stringent infection control practices are the cornerstone for curtailing the spread of the disease; and cooperation, collaboration and a united front by the global public health communities works. Interestingly, quarantining possible cases was perhaps the most effective tool for curtailing this outbreak. Still, a lot of questions remain unanswered. The most significant is why has there been such a wide range in mortality among different countries? Why do certain people act as super-spreaders? The question of period of infectiousness, age distribution, and transmissibility through routes other than droplets remains to be thoroughly investigated. Moreover, risk of transmission in households, airplanes, and ships need to be evaluated. Furthermore, there is good possibility that the SARS-CoV has been circulating and exists among exotic animals in Chinese markets.

Thus, there is a strong possibility that the virus will re-emerge during the next winter season. The most critical measures for effectively combating SARS-CoV, would be for the global healthcare communities to come together by providing better surveillance, reporting and response capability; educating healthcare workers to identify the disease; developing rapid laboratory diagnostic tests; investing in hospital pandemic plans to control nosocomial transmission and housing of large number of possible cases; community preparedness plans; and vaccine and antiviral research and development. Moreover, strict measures need to taken to control and contain the virus in the animal markets where they have been identified. Misdiagnosis of SARS cases still remains a critical issue if influenza incidence is higher during the next winter.

It remains to be seen if SARS-CoV will reemerge in the winter of 2003 but it is better to be 
proactive rather than reactive for preparing for this possible pandemic as such measures can save thousands of lives as well as an enormous global economic burden.

The Global Infectious Disease Surveillance and Alert System (GIDSAS) Program for Humanitarian Assistance, Schools of Medicine and Public Health, Johns Hopkins University, Baltimore, Maryland; Department of Emergency Medicine, Johns Hopkins Hospital, Baltimore, Maryland; Division of Health Systems, Department of International Health, Bloomberg School of Public Health, Johns Hopkins
University, Baltimore, Maryland; Center for International Emergency, Disaster and Refugee Studies, USA

\section{REFERENCES}

1 WHO. SARS website. http://www.who.int/csr/sars/ country/2003_07_04/en/.

2 WHO. SARS website. http://www.who.int/csr/don/ 2003_07_02/en/.

3 Riely S, Fraser C, Donnelly C, et al. Transmission dynamics of the etiological agent of SARS in Hong: impact of public health interventions. Science 2003;300:1961-6.

4 Lipsitch M, Cohen T, Cooper B, et al. Transmission dynamics and control of severe acute respiratory syndrome. Science 2003;300:1966-70.

\section{Learn from influenza epidemiology}

\section{Regan}

$\mathrm{T}$ he World Health Organisation announced in early July 2003 that there were then no countries where local transmission of severe acute respiratory syndrome (SARS) was continuing and that all known chains of person to person transmission have been broken. This had followed an intense period of international cooperation and aggressive public health measures since March to detect and control the spread of the disease. ${ }^{1}$ Altogether 8476 cases had been reported globally with 812 deaths. There had been some cautious optimism that such an outcome was possible even in the early phases of the outbreak and that SARS could be "driven back to nature". The observed epidemiology of this newly emerged infection suggested high virulence, particularly in the elderly population, but low transmissibility within the exception of super spreading events in hospital and some community settings. Rapid detection and isolation of suspected cases formed the back bone of control measures put in place in those countries affected, coupled with the effective implementation of infection control measures in hospitals.

We could however be merely in the eye of the storm as part of a cyclical perhaps seasonal trend in the incidence of epidemic SARS. What can we glean that may help us decide whether SARS has truly been eliminated from the human population? Has SARS merely temporarily receded into the background noise of the multitude of other respiratory infections that assail us?

We can perhaps learn from observation of the epidemiology of influenza. The influenza virus shows minor short term antigenic drift and major long term antigenic shift that are respectively typified by epidemics and pandemics. In temperate climates these short and long term cycles are overlaid on a background of distinct seasonal variation. In the northern hemisphere influenza appears to "fade out" in the early spring and summer only to return later in the autumn. Fade out is consistent with low level endemicity of the virus that falls below the threshold of detection of existing surveillance systems. ${ }^{2}$ A similar effect may be responsible for the current observed experience of SARS. It is known that the existing surveillance case definition for SARS has comparatively low sensitivity and consequently some cases will be missed. ${ }^{3}$ Until a more sensitive case definition can be agreed, supported by a specific and timely diagnostic test, a seasonal increase in SARS will not be easy to detect to focus infection control procedures and public health management. Furthermore, the animal reservoirs of SARS associated coronavirus remain and therefore the potential for the chain of events to re-occur that lead to outbreaks of SARS in China and elsewhere must be assumed to still exist.

On the balance of the current evidence it is possible that further cases of SARS will re-occur later this year riding on the tide of other seasonal respiratory viruses. How the next chapter on SARS will unfold will depend on effective targeting of improved surveillance programmes and rapid isolation of hospitalised cases. What is needed now is to translate the high level of international collaboration on research and control measures into developing more sophisticated integrated syndromic and virological surveillance systems for SARS to promptly detect and isolate suspected cases. Only then can we be sure of containing the public health and economic impact of this challenging newly emerged infection.

\section{REFERENCES}

1 World Health Organisation. Communicable disease surveillance and response. Severe acute respiratory syndrome (SARS): status of the outbreak and lessons for the immediate future. Geneva: WHO, 2003. http://www.who.int/csr/ media/sars_wha.pdf (accessed 20 May 2003).

2 Monto AS, Kiomehr F. The Tecumseh study of respiratory illness IX. Occurrence of influenza in the community 19661971. Am J Epidemiol 1975;102:553-62.

3 Rainer TH, Cameron PA, DeVilliers S, et al. Evaluation of WHO criteria for identifying patients with severe acute respiratory syndrome out of hospital: prospective observational study. BMJ 2003;326:1354-8.

\section{Debate}

M Regan, Health Protection Agency (North West), Vernon Pritchard Court, 57a Upper Northgate Street, Chester $\mathrm{CH} 1 \mathrm{4E}, \mathrm{UK}$; mregan@phls.org.uk 